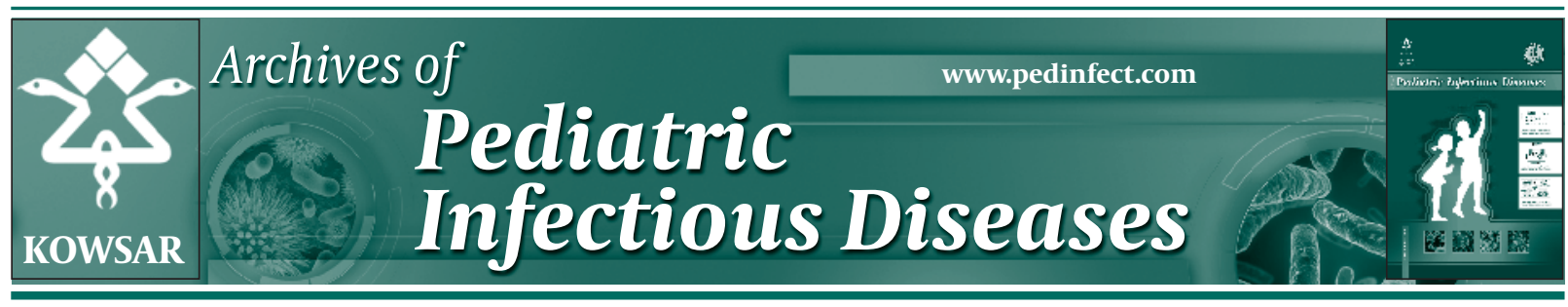

\title{
Distribution of the Pathogenicity Islands Markers (PAIs) in Uropathogenic E.coli Isolated from Children in Mofid Children Hospital
}

\author{
Masoumeh Navidinia ${ }^{1}$, Shahin Najar Peerayeh ${ }^{1,{ }^{*},}$ Fatemeh Fallah ${ }^{2}$, Bita Bakhshi ${ }^{1}$, Saadat \\ Adabian ${ }^{1}$, Shadi Alimehr ${ }^{2}$, Zari Gholinejad ${ }^{2}$ \\ ${ }^{1}$ Bacteriology Department of Medical Sciences Faculty, Tarbiat Modares University, Tehran, IR Iran \\ 2 Pediatric Infections Research Center, Shahid Beheshti University of Medical Sciences, Tehran, IR Iran \\ * Corresponding author: Shahin Najar Peerayeh, Bacteriology Department of Medical Sciences Faculty, Tarbiat Modares University, Jalal Ale Ahmad, P.O. Box \\ 14115-111, Tehran, IR Iran. Tel.: +98-2182883870, Fax: +98-2182883870, E-mail: najarp_s@modares.ac.ir
}

\section{A B S T R A C T}

Background: Uropathogenic Escherichia coli (UPEC) is a causative agent of most of urinary tract infections (UTIs), which expresses a multitude of virulence factors.

Objectives: The aim of this study was to investigate virulence associated characteristics characteristics in UPEC isolates derived from urine specimens, and to investigate the distribution of the pathogenicity islands virulence markers (PAIs) among the isolates in relation to their antibiotic susceptibility pattern.

Patients and Methods: A total of 50 E.coli isolates were collected from patients with UTI during September 2009 to August 2010. Biochemical and standard microbiological techniques were used to identify E.coli followed by screening for islands by polymerase chain reaction (PCR).

Results: We found a high number of PAI markers such as PAI ICFT073, PAI IICFT073, PAI I536, PAI IV536, PAI II J96, and PAI II536 significantly associated with UPEC. High level of resistance to Aztreonam, Co-trimoxazole, Cefpodoxime, and Cefotaxime was found among the UPEC isolates.

Conclusions: This observation is of special value considering that the UPEC pathotype constitutes an emerging group of enteropathogens, particularly, in our county. Knowledge of the molecular details of Uropathogenic E.coli is useful to develop successful strategies for the treatment of urinary tract infection and complications associated with UTIs in human.

Keywords: Escherichia coli; Urinary Tract Infection; Genomic Islands

Copyright () 2013, Pediatric Infections Research Center

Article type: Research Article; Received: 11 Nov 2012, Revised: 29 Nov 2012, Accepted: 17 Dec 2012; DOI: 10.5812/pedinfect.9083

-Implication for health policy/practice/research/medical education:

Knowldge of the molecular details of Uropathogenic E.coli is useful in developing successful strategies for the treatment of urinary tract infection and complications associated with UTIs in human.

-Please cite this paper as:

Navidinia M, Najar Peerayeh S, Fallah F, Bakhshi B, Adabian S, Alimehr S, et al. Distribution of Pathogenicity Islands Markers (PAIs) in Uropathogenic E.coli Isolated from Children in Mofid Children Hospital.Arch Pediatr Infect Dis.2013;1(2): 75-79. DOI: 10.5812/pedinfect.9083 


\section{Background}

Urinary tract infections (UTIs) currently rank among the most prevalent infectious diseases worldwide, with chronic and recurrent infections being especially problematic ( 1 , 2). The primary etiologic agents associated with UTIs are strains of Uropathogenic Escherichia coli (UPEC) (3). UPEC strains express a wide spectrum of virulence factors to colonize urinary tract of mammalian. UPEC can invade some host cell types, such as the terminally differentiated superficial facet cells and less mature intermediate and basal epithelial cells and comprise the stratified layers of the bladder urothelium. To facilitate both the establishment and persistence of UPEC within the urinary tract, host cell invasion is needed $(4,5)$ Moreover, pathogenic extraintestinal isolates harbour specialised virulence factors, i.e., traits that confer pathogenic potential, which are infrequent among commensal isolates (2).

Moreover, extraintestinal E.coli isolates harbor some virulence Factors that are infrequent among commensal E.coli isolates. Some of these virulence factors are usually encoded on the pathogenicity islands (PAIs), providing a mechanism for coordinated horizontal factors, i.e., traits that confer pathogenic virulence genes transferation (6).

Hacker et al. (7) first described PAI concept in the late 1980s. These mobile genetic elements comprise short direct repeats of fragments of DNA $(>10 \mathrm{~kb})$ adjacent to the tRNA genes and contain insertion sequences, integrases and transposases, and have a $\mathrm{G}+\mathrm{C}$ content that differs from the host bacterial genome (8).

\section{Objectives}

The aim of this study was to investigate virulence associated characteristics in UPEC isolates derived from urine specimens, and to investigate the distribution of the pathogenicity islands virulence markers (PAIs) among the isolates in relation to their antibiotic susceptibility pattern.

\section{Patients and Methods}

\subsection{Specimens and Patients}

A total of 50 E.coli isolates from urinary specimens were collected from patients with UTI, between 2-12 years old and admitted to Mofid children hospital, Tehran, Iran, during September 2009 to August 2010.

Samples were derived from fresh midstream urine, cultured $(0.01 \mathrm{~mL})$ on MacConkey agar as well as Sheep blood agar and incubated at $37^{\circ} \mathrm{C}$ for 24 hours. Cultures with more than $10^{5} \mathrm{CFU} / \mathrm{mL}$ were examined for further analysis. Susceptibility to Nitrofurantoin $(300 \mu \mathrm{g})$, Ciprofloxacin $(5 \mu \mathrm{g})$, Nalidixic acid (30 $\mu \mathrm{g})$, Amoxicillin $(10 \mu \mathrm{g})$, Amoxiclave $(30 \mu \mathrm{g})$, Gentamicin $(120 \mu \mathrm{g})$, Ceftazidime (30 $\mu \mathrm{g})$, Cefpodoxime $(10 \mu \mathrm{g})$, Aztreonam $(30 \mu \mathrm{g})$, Imipenem $(10 \mu \mathrm{g})$, Amikacin $(30 \mu \mathrm{g})$, Norfloxacin $(10 \mu \mathrm{g})$, Co-trimoxazole $(25 \mu \mathrm{g})$ and Cefotaxime $(30 \mu \mathrm{g})$ were determined by disc diffusion based on CLSI criteria (nonfastidious groupings M2-disk diffusion M100) (2). The intermediate susceptibility was considered as susceptible (9).

\subsection{DNA Extraction}

The DNA was extracted according to the protocol described by Radhuoni et al. in 2009. The isolates were cultured on MacConkey agar plates for $24 \mathrm{~h}$. One to two colonies from pure cultures were resuspended in $0.5 \mathrm{~mL}$ sterile distilled water. The cells were lysed by heating at $950 \mathrm{C}$ for $10 \mathrm{~min}$ and the supernatant was harvested by centrifugation at 12,000 rpm (8000g) for $5 \mathrm{~min}$. The supernatant was used as the source of the template DNA (10).

\subsection{PCR Components and Amplification Profile}

Briefly, PCR was performed in a reaction mixture containing a $300 \mathrm{nM}$ concentration of each oligonucleotide primer (Bioneer); $5.5 \mathrm{mM} \mathrm{MgCl}$; $200 \mathrm{mM}$ (each) deoxynucleoside triphosphates and $0.125 \mathrm{U}$ of Taq DNA polymerase (from Genetbio).

Amplification profile was $94^{\circ} \mathrm{C}$ for $5 \mathrm{~min}$, followed by 30 cycles of $94^{\circ} \mathrm{C}$ for $1 \mathrm{~min}, 55^{\circ} \mathrm{C}$ for $1 \mathrm{~min}$ and $72^{\circ} \mathrm{C}$ for $1 \mathrm{~min}$, with a final extension step at $72^{\circ} \mathrm{C}$ for $10 \mathrm{~min}$.

\subsection{Detection of PAI Markers by Multiplex PCR}

All isolates were screened for the presence of PAI markers (PAI ICFT073, PAI IICFT073, PAI I536, PAI II536, PAI III536, PAI IV536, PAI I J96, and PAI II J96) by using the PCR method. Primers used in this study are shown in Table 1.

\section{Results}

A total of 50 E.coli isolates were analyzed from 50 patients with UTI who have admitted to the nephrology ward of Mofid children hospital, Tehran, Iran. Urine samples isolated from patients of both sexes (85\% girl, 17.5\% boy) aged between 2-12 years.

\subsection{Pattern of Resistance Among Escherichia coli Isolates}

High resistance to Aztreonam, Co-trimoxazole, Cefpodoxime, and Cefotaxime were found among UPEC isolates. Resistance pattern of UPEC isolates were shown in Figure 1. Multidrug resistance which was defined as resistance to more than 3 antibiotics was most commonly observed in UPEC isolates (26\%).

\subsection{Pathogenicity Island Genes Distribution}

Distribution of the pathogenicity island genes in UPEC is as follows: In total, $89 \%$ of UPEC isolates contained PAIs. The PAI IV536 (86\%) were PAI ICFT073 (74\%) isolates were the most among the UPEC E.coli, whereas PAI III536 (6\%) and PAI I J96 (4\%) were the least (Figure 2). 
Table 1. Primer Sequences Were Used in This study

\begin{tabular}{|c|c|c|}
\hline Reference & Primer & BP Gene \\
\hline DobrindtU, etal.(22) & CGGGCATGCATCAATTATCTTTGTGTGTAGATGCAGTCACTCCG & 200 PAI III536 \\
\hline Karch H, (8) & AAGGATTCGCTGTTACCGGACTCGTCGGGCAGCGTTTCTTCT & 300 PAI IV536 \\
\hline M. Sabate, (2) & ATGGATGTTGTATCGCGCPSACGAGCATGTGGATCTGC & 400 PAI IICFT073 \\
\hline M. Sabate, (2) & TAATGCCGGAGATTCATTGTCAGGATTTGTCTCAGGGCTTT & 1800 PAI I536 \\
\hline M. Sabate, (2) & CCATGTCCAAAGCTCGAGCCTACGTCAGGCTGGCTTTG & 1000 PAI II 536 \\
\hline Johnson JR, (4) & TCGTGCTCAGGTCCGGAATTTTGGCATCCCACATTATCG & 400 PAI I J96 \\
\hline Landraud L, 2003 & GGATCCATGAAAACATGGTTAATGGGGATATTTTTGTTGCCATTGGTTACC & 2300 PAI II J96 \\
\hline Johnson JR, (4) & GGACATCCTGTTACAGCACGCATCGCCACCAATCACAGCCGAAC & 930 PAI ICFT073 \\
\hline
\end{tabular}

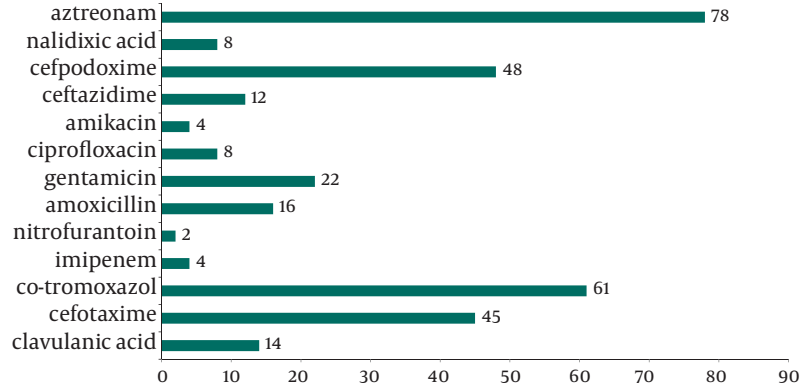

Figure 1. The Prevalence of Antibiotic Resistance Pattern in Uropathogenic Isolates Obtained from Children With UTI Antibiotics \% Resistance

\subsection{Statistical Analysis}

Statistical analysis was performed by using the Fisher exact and chi-square tests. P value $<0.05$ was considered as statistically significant.

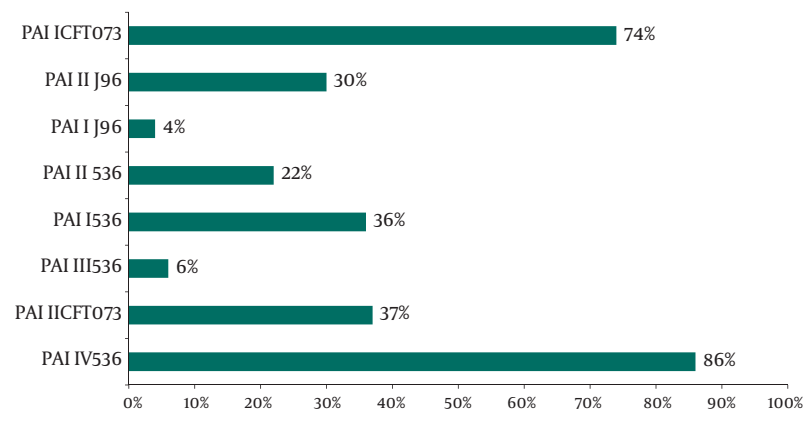

Figure 2. The Pathogenicity Island Marker Genes Distribution in UPEC Isolates From Patients With UTI

\section{Discussion}

UTI is usually treated empirically without culturing the urine sample but this type of infection accounts for about $10 \%$ to $15 \%$ prolongation of hospitalization due to the emergence of resistance to antibiotics among the causative bacteria, particularly among UPEC strains. This may result in the spread of antibiotic resistant bacteria which led us to investigate this possibility by testing the effects of a range of functionally distinct antibiotics on UPEC to reduce morbidity and the duration of hospitalization in patients with UTI (11). Accordingly, we considered E.coli strains isolated from urine and examined pattern of antibiotic resistance and pathogenicity islands.

Resistance to several antibiotics was observed in UPEC isolates. All isolates were highly resistant to trimethoprim-sulfamethoxazole (61\% in UPEC) which is a widely used antibiotic for UTI treatment, indicating that it has become nearly ineffective for UTI treatment, however, a higher rate to Azteronam was also found among the isolates.

Blango in 2010 (1) 267 demonstrated that resistance to Amoxicillin, Gentamicin and Co-trimoxazole E.coli isolates was seen in $45.1 \%, 72 \%$ and $78.4 \%$ of E.coli strains, whereas in this study, a different resistance pattern was obsereved (Figure 1). Cephalosporins, Cefpodoxime and Cefotaxime were less effective in UTI treatment than other antibiotics regardless of E.coli phylogenetic group (You haven't speak about phylogenetic groups, previously). In our study, a high rate of resistance to Co-trimoxazole and sensitivity to Imipenem was observed, which is in agreement with the finding of Farshad et al. (year, country) (12). In a research by Moreno et al. (13) found 21\% resistance to quinolone antimicrobials and $18 \%$ to fluoroquinolones, whereas Matthew et al. (14) demonstrated that only one E.coli isolate was resistant to Ciprofloxacin. Recently, Shigemura et al. (15) has reported an emergence of fluoroquinolone resistant E.coli responsible for UTIs, however in this study a higher resistance to quinolones (8\%) was observed.

In our study, a lower resistance to Ciprofloxacin, Nalidixic acid, Gentamicin, Trimethoprim Sulfamethoxazole was found compared to the Alhaj et al. research in Malaysia 2007 (16), meanwhile, resistance to Ceftazidime (11\%) and Amoxicillin-clavulanic acid (17\%) was consistent with results of Bruzuskiewicz et al. in 2006 (17). In this study, resistance to Amikacin (6\%) was relatively lower than re- 
sults of Bingen-Bidois in 2002 (27\%) (18) and Parham in $2005(22.4 \%)$ (19).

Murugan etal. in 2012, studied antibiotic resistance among uropathogens from rural teaching hospital. The predominant Gram negative uropathogenic E. coli $(\mathrm{n}=44)$ showed resistance to Nitrofurantoin (1.5\%), Amikacin (10.5\%), Gentamicin (30.4\%), Ciprofloxacin (70.4\%), Cefpodoxime (72.6\%), Cefotaxime (74.8\%), Nalidixic acid (98.5\%). The E.coli shows higher susceptibility (about 90\%) only to nitrofurantoin and amikacin was presented. All strains was more resistant than compared with our research. The isolated uropathogens antibiotic resistance on the whole observed in this study is higher than the our results from urine which is an alarming one (20).

Herzer et al. (21) reported that pathogenicity islands (PAIs) were enriched among E.coli and caused extraintestinal infections. Here, we also confirmed the prevalence of eight PAI markers in E.coli strains isolated from the urine of children with UTI.

Dobrindt et al. (22) in 2002 studied genetic structure and distribution of four pathogenicity islands (PAI I536 to PAI IV536) of Uropathogenic Escherichia coli strain 536. In Uropathogenic E.coli, multiple PAI sequences (PAI I536 to PAI IV536) were studied and their presence in several wildtype E.coli isolates was extensively investigated. Their results suggested PAIs are detectable in pathogenic E.coli isolates. We determined that the acquisition of large DNA regions, such as PAIs, was an important factor in the evolution of bacterial pathogens. Sabate et al. (2) in 2006 studied the pathogenicity island markers in commensal and Uropathogenic Escherichia coli isolates. Their results showed the mean number of PAIs per isolate was high among UPEC

Similar with Brzuszkiewicz et al. results (18) genomic differences between UPEC strains were mainly restricted to large pathogenicity islands.

It seems reasonable that UPEC might show similar adaptations for an extraintestinal lifestyle, which, in turn, enables them to cause extraintestinal disease in humans. Presentation of the molecular details of UPEC is necessary to develop successful strategies for prevention of human urinary tract infections and urological complications associated with UTIs.

\section{Acknowledgements}

This work was supported by Tarbiat Modares University and Pediatric Infection Research

\section{Authors' Contribution}

None declared.

\section{Financial Disclosure}

There was no financial support for this study.

\section{Funding/Support}

None declared.

\section{Ethical Considerations}

This study was performed using routine samples obtained from patients admitted to Mofid Children Hospital and thus ethical approval was not required.

\section{References}

1. Blango MG, Mulvey MA. Persistence of uropathogenic Escherichia coli in the face of multiple antibiotics. Antimicrob Agents Che mother. 2010;54(5):1855-63.

2. Sabate M, Moreno E, Perez T, Andreu A, Prats G. Pathogenicity island markers in commensal and uropathogenic Escherichia coli isolates. Clin Microbiol Infect. 2006;12(9):880-6.

3. Sivick KE, Mobley HL. Waging war against uropathogenic Escherichia coli: winning back the urinary tract. Infect Immun. 2010;78(2):568-85.

4. Johnson JR, Scheutz F, Ulleryd P, Kuskowski MA, O'Bryan TT, Sandberg T. Phylogenetic and pathotypic comparison of concurren urine and rectal Escherichia coli isolates from men with febrile urinary tract infection. J Clin Microbiol. 2005;43(8):3895-900.

5. Mulvey MA, Schilling JD, Martinez JJ, Hultgren SJ. Bad bugs and beleaguered bladders: interplay between uropathogenic Escherichia coli and innate host defenses. Proc Natl Acad Sci U S A. 2000;97(16):8829-35

6. Hacker J, Kaper JB. Pathogenicity islands and the evolution of microbes. Annu Rev Microbiol. 2000;54:641-79.

7. Hacker J, Bender L, Ott M, Wingender J, Lund B, Marre R, et al. Deletions of chromosomal regions coding for fimbriae and hemolysins occur in vitro and in vivo in various extraintestinal Escherichia coli isolates. Microb Pathog. 1990;8(3):213-25.

8. Karch H, Schubert S, Zhang D, Zhang W, Schmidt H, Olschlager $\mathrm{T}$, et al. A genomic island, termed high-pathogenicity island, is present in certain non-O157 Shiga toxin-producing Escherichia coli clonal lineages. Infect Immun. 1999;67(11):5994-6001.

9. Schlager TA, Hendley JO, Bell AL, Whittam TS. Clonal diversity of Escherichia coli colonizing stools and urinary tracts of young girls. Infect Immun. 2002;70(3):1225-9.

10. Sabarinath A, Deallie C, Belot G, Vanpee G, Matthew V, Sharma $\mathrm{R}$, et al. Antimicrobial Resistance And Phylogenetic Groups Of Commensal Escherichia Coli Isolates From Healthy Pigs In Grenada. Webmed Central. 2011;WMC001942.

11. Rodriguez-Siek KE, Giddings CW, Doetkott C, Johnson TJ, Fakhr MK, Nolan LK. Comparison of Escherichia coli isolates implicated in human urinary tract infection and avian colibacillosis. Microbiology. 2005;151(Pt 6):2097-110.

12. Farshad S, Japoni A, Hosseini M. Low distribution of integrons among multidrug resistant E. coli strains isolated from children with community-acquired urinary tract infections in Shiraz, Iran. Pol J Microbiol. 2008;57(3):193-8.

13. Moreno E, Prats G, Sabate M, Perez T, Johnson JR, Andreu A. Quinolone, fluoroquinolone and trimethoprim/sulfamethoxazole resistance in relation to virulence determinants and phylogenetic background among uropathogenic Escherichia coli. J Antimicrob Chemother. 2006;57(2):204-11.

14. Tager Frey M, Vial PC, Castillo CH, Godoy PM, Hjelle B, Ferres MG Hantavirus prevalence in the IX Region of Chile. Emerg Infect Dis. 2003;9(7):827-32.

15. Shigemura K, Arakawa S, Miura T, Nakano Y, Tanaka K, Fujisawa M. Significance of fluoroquinolone-resistant Escherichia coli in urinary tract infections. Jpn J Infect Dis. 2008;61(3):226-8.

16. Alhaj N, Mariana N, Raha A, Ishak Z. Prevalence of antibiotic resistance among Escherichia coli from different sources in Malaysia. Int J Poultry Sci. 2007;6(4):293-7.

17. Brzuszkiewicz E, Bruggemann $H$, Liesegang $H$, Emmerth $M$, Olschlager T, Nagy G, et al. How to become a uropathogen: comparative genomic analysis of extraintestinal pathogenic Esch- 
erichia coli strains. Proc Natl Acad Sci US A. 2006;103(34):12879-84.

18. Bingen-Bidois $\mathrm{M}$, Clermont $\mathrm{O}$, Bonacorsi S, Terki M, Brahimi N, Loukil C, et al. Phylogenetic analysis and prevalence of urosepsis strains of Escherichia coli bearing pathogenicity island-like domains. Infect Immun. 2002;70(6):3216-26.

19. Parham NJ, Pollard SJ, Chaudhuri RR, Beatson SA, Desvaux M, Russell MA, et al. Prevalence of pathogenicity island IICFT073 genes among extraintestinal clinical isolates of Escherichia coli. J Clin Microbiol. 2005;43(5):2425-34.

20. Murugan K, Savitha T, Vasanthi S. Retrospective study of antibi- otic resistance among uropathogens from rural teaching hospital, Tamilnadu, India. Asian Pacific J Tropical Dis. 2012;2(5):375-80.

21. Herzer PJ,Inouye S, Inouye M, Whittam TS. Phylogenetic distribution of branched RNA-linked multicopy single-stranded DNA among natural isolates of Escherichia coli. J Bacteriol.1990;172(11):6175-81.

22. Dobrindt U, Blum-Oehler G, Nagy G, Schneider G, Johann A, Gottschalk G, et al. Genetic structure and distribution of four pathogenicity islands (PAI I(536) to PAI IV(536)) of uropathogenic Escherichia coli strain 536. Infect Immun. 2002;70(11):6365-72. 